TRANSACTIONS OF THE

AMERICAN MATHEMATICAL SOCIETY

Volume 354, Number 4, Pages 1631-1650

S 0002-9947(01)02919-1

Article electronically published on November 19, 2001

\title{
EXTREMAL PROBLEMS FOR QUASICONFORMAL MAPS OF PUNCTURED PLANE DOMAINS
}

\author{
VLADIMIR MARKOVIĆ
}

\begin{abstract}
The main goal of this paper is to give an affirmative answer to the long-standing conjecture which asserts that the affine map is a uniquely extremal quasiconformal map in the Teichmüller space of the complex plane punctured at the integer lattice points. In addition we derive a corollary related to the geometry of the corresponding Teichmüller space. Besides that we consider the classical dual extremal problem which naturally arises in the tangent space of the Teichmüller space. In particular we prove the uniqueness of Hahn-Banach extension of the associated linear functional given on the Bergman space of the integer lattice domain. Several useful estimates related to the local and global properties of integrable meromorphic functions and the delta functional (see the definition below) are also obtained. These estimates are intended to study the behavior of integrable functions near singularities and they are valid in general settings.
\end{abstract}

\section{INTRODUCTION}

Let $M$ be the hyperbolic Riemann surface and let Teich $(M)$ denote the Teichmueller space of the surface $M$. Each point in Teich $(M)$ can be represented in the following way (see [6], [14]). Let $f$ denote a quasiconformal map of the surface $M$ onto another Riemann surface $N$. Denote by $[f]$ the class of all quasiconformal maps defined on $M$ such that the quasiconformal map $g$ belongs to $[f]$ if and only if the composition $f \circ g^{-1}$ is homotopic to a conformal map. We say that $[f]$ is an element of Teich $(M)$. It is well known (see [6],[14]) that the Teichmueller space is a complex manifold with the naturally defined complex structure coming from the Bers emmbending.

Denote by $A^{1}(M)$ the complex Banach space of all integrable holomorphic quadratic differentials on $M$. The space $A^{1}(M)$ is also called the Bergman space of the surface $M$. The tangent space of Teich $(M)$ at the origin $[i d]$ of $T e i c h(M)$ is isomorphic to the Banach dual of $A^{1}(M)$. Denote by $L^{1}(M)$ and $L^{\infty}(M)$ respectively the complex Banach spaces of all integrable quadratic differentials on $M$ and all essentially bounded $(-1,1)$ complex forms on $M$. It is well known that $L^{\infty}(M)$ is the Banach dual of $L^{1}(M)$. Since $A^{1}(M)$ is a subspace of $L^{1}(M)$ it follows that every bounded linear functional on $A^{1}(M)$ can be represented by some $\mu \in L^{\infty}(M)$. Since this choice is not unique we denote by $[\mu]^{*}$ the class of all elements of $L^{\infty}(M)$ representing the same linear functional as $\mu$.

Received by the editors March 23, 2000.

1991 Mathematics Subject Classification. Primary 41A65, 30C75.

(C)2001 American Mathematical Society 
Recall that the map $g \in[f]$ is said to be an extremal quasiconformal map in $[f]$ if the following condition is satisfied:

$$
\|\operatorname{Belt}(g)\|_{\infty}=\inf _{h \in F(f)}\|\operatorname{Belt}(h)\|_{\infty},
$$

where $\operatorname{Belt}(g)=\frac{g_{\bar{z}}}{g_{z}}$ is the Beltrami differential of $g$. The map $g$ is uniquely extremal in $[f]$ if there are no other extremal maps in $[f]$. The classical extremal problem, first studied by Grötzsch and Teichmueller, is to determine whether the class $[f]$ contains a uniquely extremal map (for example, see [6],[14, [18, [21]). Let

$$
Z=\left\{z_{k, l}: z_{k, l}=k+i l, k, l \in \mathbf{Z}\right\}
$$

be the integer lattice in the complex plane, and

$$
S=\mathbf{C} \backslash Z
$$

the corresponding plane domain. If $K>1$, let

$$
A_{K}(z)=K x+i y
$$

denote the affine map of the complex plane. Denote by $\left[A_{K}\right]$ the corresponding element of Teich $(S)$. The main purpose of this paper is to give an affirmative answer to the following well known conjecture posed for the first time in [19.

Conjecture 1 (Reich, Strebel). The affine map $A_{K}$ is a uniquely extremal quasiconformal map in its class $\left[A_{K}\right]$.

Our main result is

Theorem 1.1. The affine map of the domain $S$ is uniquely extremal in the class $\left[A_{K}\right]$.

In their paper [19], E.Reich and K.Strebel proved that $A_{K}$ is extremal in $\left[A_{K}\right]$ answering the question of I.Kra. Moreover, they characterized all punctured plane domains where the affine maps are extremal. The corresponding question for unique extremality seems to be more difficult.

The lattice domain $S$ has certain important symmetry properties. To illustrate them we observe the following. The domain $S$ is invariant under translation for vectors $z_{k, l} \in Z$. Therefore $S$ is a natural covering surface of the once punctured torus $\mathbf{T}$. The corresponding complex structure on $\mathbf{T}$ is uniquely determined by the choice of the covering group $G$. The group $G$ is generated by the translations $z+1$ and $z+i$. Affine map $A_{K}$ preserves the structure of the torus and therefore represents the element of the $T \operatorname{eich}(\mathbf{T})$ itself. The group of covering transformations is isomorphic to $\mathbf{Z} \oplus \mathbf{Z}$. It interesting to note that this group is amenable (see [13]). By the results of C.McMullen (see [13]) we conclude that the classical Poincare Theta operator $\Theta: A^{1}(S) \rightarrow A^{1}(\mathbf{T})$ has the Banach norm equal to one. The Banach space $A^{1}(\mathbf{T})$ is of the dimension one. By the application of the well known Hamilton-Krushkal-Reich-Strebel for extremality, one concludes that the affine map $A_{K}$ is an extremal quasiconformal map. This observation provides an alternative proof for extremality of the affine map of the domain $S$. This is also related to certain similar problems treated by K.Strebel, C.McMullen, I.Kra (for an example see [13], [21], [22]).

So we have demonstrated that the lattice domain has some nice properties closely related to the fact that $A_{K}$ is extremal. But concerning the question of unique extremality it seems that the essential fact which implies the unique extremality is the 
distribution of the set $Z$ near infinity in the complex plane. Our proof makes use of algebraic and geometric lattice properties, but since we believe that the distribution of the points is essential we conjecture the following possible generalization of our result:

Conjecture 2. Let $P$ be an infinite set of points in the complex plane with the property that for any given disc of Euclidean radius one in the complex plane, one can find point $p \in P$ which belongs to the given disc. Then the affine map of the domain $\mathbf{C} \backslash P$ is uniquely extremal.

There exist several important characterizations of the unique extremality in terms of the geometry of Teichmueller space Teich $(M)$ of Riemann surface $M$ (see 5]). In particular if $M$ is a plane domain, then the affine map $A_{K}$ is a uniquely extremal quasiconformal map of $M$ if there exists a unique normalized isometry of the unit disc into the Teichmueller space of the plane domain $M$. There exists special interest in studying the isometries of the unit disc into the Teichmueller space of punctured plane domains (see [2]). We state the following corollary of Theorem 1.1. Its proof follows directly from Theorem 1.1 and Theorem 6 in [5].

Corollary 1. There exists a unique isometry $f$ of the unit disc $\Delta$ into the Teich $(S)$ with the following properties: $1^{\circ} f(0)=[0]$ and $2^{\circ} f^{\prime}(0)=[1]^{*}$.

We now recall the recent results which have provided the complete analytic characterization of uniquely extremal quasiconformal maps. Then we state the corollary of Theorem 1.1 related to the classical dual extremal problem related to the uniqueness of Hahn-Banach extensions. Recall that each bounded linear functional $\lambda$ on $L^{1}(M)$ is uniquely represented as

$$
\lambda(\phi)=\int_{M} \operatorname{Re}(\phi \mu), \quad \phi \in L^{1}(M),
$$

where $\mu$ is a fixed element of $L^{\infty}(M)$. Suppose that $f: M \rightarrow N$ is a uniquely extremal quasiconformal map. Set $\mu=\operatorname{Belt}(f)$. One of the two main results in [3] (see also [4, 11], 10, 12]) is:

Proposition 1.1. The mapping $f$ is a uniquely extremal quasiconformal map if and only if the linear functional $\lambda: A^{1}(M) \rightarrow \mathbf{C}$ given by

$$
\lambda(\phi)=\int_{M} \operatorname{Re}(\phi \mu), \quad \phi \in A^{1}(M),
$$

has a unique Hahn-Banach extension to $L^{1}(M)$.

The linear functional $\lambda$ is also said to be $H B U$ (abbreviation for Hahn-Banch unique) if it has a unique Hahn-Banach extension from $A^{1}(M)$ onto $L^{1}(\mathbf{M})$. This proposition gives the necessary and sufficient conditions for the map $f$ to be uniquely extremal. It also represents a generalization of the well known characterization of extremal quasiconformal maps obtained by Reich, Strebel, Hamilton, Krushkal (see [6], [8], [14, [18, [21]). From Theorem 1.1 and Proposition 1.1 we derive the following corollary:

Corollary 2. Let $\lambda$ denote the linear functional on $A^{1}(S)$ given by

$$
\lambda(\phi)=\operatorname{Re} \int_{\mathbf{C}} \phi
$$


Then the linear functional $\lambda$ has a unique Hahn-Banach extension onto the space $L^{1}(\mathbf{C})$.

In order to prove Theorem 1.1 we do not work directly with the quasiconformal maps of the integer lattice domain $S$. The following proposition is due to E.Reich (see [16]). It gives sufficient conditions for the affine map to be uniquely extremal. With the notation stated above we have

Proposition 1.2. Let $M$ be a plane domain. The affine map $A_{K}$ is a uniquely extremal quasiconformal map in $\left[A_{K}\right]$ if there exists a sequence $\left\{\phi_{n}\right\}, \phi_{n} \in A^{1}(M)$ and a constant $C$ with the following properties:

$1^{\circ}$

$$
\delta\left(\phi_{n}\right)=\int\left|\phi_{n}\right|-\operatorname{Re}\left(\phi_{n}\right)=\delta\left(\phi_{n}\right)<C, \quad n \in \mathbf{N} .
$$

$2^{\circ}$

$$
\lim _{n \rightarrow \infty} \phi_{n}(p)=1, \quad p \in M .
$$

$3^{\circ}$ If $\Omega_{K, n}=\left\{z \in M:\left|\phi_{n}(z)\right|>K\right\}$, then we have

$$
\lim _{K \rightarrow \infty} \int_{\Omega_{K, n}}\left|\phi_{n}\right|=0
$$

uniformly in $n \in \mathbf{N}$.

It is known (see [3, [4, 10, 12]) that the affine map is uniquely extremal if and only if there is a sequence $\phi_{n}$ from $A^{1}(M)$ which satisfies the first two conditions of Proposition 1.2 and that in particular $\delta\left(\phi_{n}\right) \rightarrow 0$ when $n \rightarrow \infty$. Although this last statement provides the necessary and sufficient conditions it is usually easier to construct a sequence where the $\delta$ functional is bounded and the third condition of the above proposition is satisfied rather then a sequence where the delta functional tends to zero. On the other hand it is known to be hard to construct such sequences and to date there are only a few such examples (for an example see [16]). We will explicitly construct the corresponding sequence which satisfies the conditions of Proposition 1.2 and where the corresponding plane domain $M$ is the lattice domain $S$. The remainder of the paper is organized as follows. In Section 2 by using the elementary properties of the suitably chosen elliptic function we introduce representation of functions from $A^{1}(S)$ (in the remainder of this paper we write just $\left.A^{1}\right)$. In Section 3 by using this representation result from Section 2 we construct the sequence $\left\{\phi_{n}\right\}$ which satisfies the first two assumptions of Proposition 1.2. In Section 4 we complete the proof of Theorem 1.1 by proving that that the sequence $\left\{\phi_{n}\right\}$ we construct in Section 3 satisfies the third assumption of Proposition 1.2.

Acknowledgment. I am thankful to V.Božin and E.Reich for reading the manuscript and for their valuable suggestions.

\section{RePRESENTATion of FUnCTIONS From $A^{1}$}

The function $\phi \in A^{1}$ is a meromorphic function with at most the first order poles at the lattice points $z_{k, l} \in Z$. Denote by $\lambda_{k, l}$ the residue of $\phi(z)$ at the point 
$z_{k, l}$. Since $\phi(z)$ is an integrable function in the complex plane we find that $\phi(z)$ is uniquely determined by $\lambda_{k, l}$ and moreover it admits the representation

$$
\phi(z)=\sum_{k, l} \frac{\lambda_{k, l}}{z-z_{k, l}}
$$

for $z \in S$. But in order to construct the sequence which satisfies the assumptions of Proposition 1.2, it is not convenient to use the above representation. In this section we prove that there exists a suitable representation which applies to certain functions $\phi \in A^{1}$. Let $W$ denote another integer lattice in the complex plane, obtained by translating the lattice $Z$ by the vector $\frac{1+i}{2}$. That is, we have

$$
W=\left\{w_{k, l}: w_{k, l}=k+\frac{1}{2}+i\left(l+\frac{1}{2}\right), k, l \in \mathbf{Z}\right\} .
$$

While $Z$ represents the part of boundary of the domain $S$ we have that $W$ is a subset of $S$. Set $\phi\left(w_{k, l}\right)=\alpha_{k, l}, k, l \in \mathbf{Z}$. From the mean value property theorem for analytic functions it follows that the double sequence $\alpha_{k, l}$ is absolutely integrable, that is we have

$$
\sum_{k, l}\left|\alpha_{k, l}\right|<\infty
$$

On the other hand the following question naturally arises. Whether for every absolutely integrable double sequence $\alpha_{k, l}$ there exists a function $\phi \in A^{1}$ such that $\phi\left(w_{k, l}\right)=\alpha_{k, l}$. In general the answer is negative as we will soon see. The objective of this section is to show that under additional assumptions on the double sequence $\alpha_{k, l}$ we can explicitly construct function $\phi \in A^{1}$ which satisfies the above assumption. The corresponding result is given by Lemma 2.1 .

Let us recall a few facts from the theory of elliptic functions (see [1, [9]). There exists a unique meromorphic function $P(z)$ in the complex plane with the following properties:

$1^{\circ} P(z)=-P(z+1)=-P(z+i)$.

$2^{o} P(z)=\left(z-w_{0,0}\right)+o\left(z-w_{0,0}\right)$, for $z$ near $w_{0,0}$.

$3^{\circ} P(z)$ has only the first order poles at the point $z_{k, l} \in Z$.

The function $P(z)$ is the normalization of the classical Jacobian eliptic cosine function. The function $P(z)$ can also be understood in the following way. Set $T_{1}(z)=z+(1-i)$ and $T_{2}(z)=z+(1+i)$ and let $\Gamma$ be the Abelian group generated by $T_{1}$ and $T_{2}$. Then $S / \Gamma$ is the well defined Riemann surface conformally equivalent to the twice punctured torus. There are only two linearly independent integrable holomorphic quadratic differentials supported on $S / \Gamma$. One of them is represented by the constant function and the other one is represented by the function $P(z)$.

The periodicity of $P(z)$ further yields the following conclusions (see [1, 9]) about zeroes and poles of the function $P(z) . P(z)$ has the first order poles at the points $z_{k, l}$. The residues of $P(z)$ at the points $z_{k, l}$ are uniquely determined by value of the residue of $P(z)$ at the point $z_{o, o}$ which can be expressed as the value of certain special function (see [1]). But in this paper we do not need the exact values of these residues because we only use the fact that $P(z)$ has the first order poles at the points $z_{k, l}$. On the other hand $P(z)$ has the first order zeroes at the points $w_{k, l}$ and the function $P(z)$ admits the following representation near the point $w_{k, l}$ :

$$
P(z)=(-1)^{(k+l)}\left(z-w_{k, l}\right)+o\left(z-w_{k, l}\right) .
$$


Next, we define the connected domain $S_{1}$ by

$$
S_{1}=\left\{z \in S: \min _{k, l \in \mathbf{Z}}\left|z-z_{k, l}\right|>\frac{1}{8}\right\} .
$$

The function $P(z)$ is analytic on $S_{1}$. From the periodicity of $P(z)$ (also one may understand $S_{1} / \Gamma$ as a compact subset of the above mentioned twice punctured torus) and the fact that $P(z)$ has the zeroes at the points $w_{k, l}$ we conclude that there is a constant $C_{1}>0$ such that the estimates

$$
|P(z)| \leq C_{1},
$$

$$
\frac{|P(z)|}{\left|z-w_{k, l}\right|} \leq C_{1}, \quad z \in S_{1}
$$

hold when $z \in S_{1}$.

Suppose that $\alpha$ is a complex number and $(p, q)$ an arbitrary pair of integers. Set

$$
\phi(z)=\frac{(-1)^{(p+q)} \alpha}{z-w_{p, q}} P(z) .
$$

The function $\phi(z)$ satisfies

$$
\phi\left(w_{k, l}\right)=0, \quad(k, l) \neq(p, q)
$$

and $\phi\left(w_{p, q}\right)=\alpha$. It is easy to see that $\phi$ does not belong to $A^{1}$ because it behaves as $O\left(\frac{1}{|z|}\right)$ when $z \in S_{1}$ and $z \rightarrow \infty$. We conclude from (3) and (4) that if one takes the double sequence $\alpha_{k, l}$ such that only $\alpha_{p, q}=\alpha$ is different than zero, then this double sequence is absolutely integrable but the corresponding meromorphic function $\phi$ is not. On the other hand, let $\alpha_{k, l}$ be an arbitrary double sequence of complex numbers. By replacing $\alpha=\alpha_{k, l}$ in (4) for each pair $(k, l)$ and by summing over $k, l \in \mathbf{Z}$ we formally obtain the following sum:

$$
\phi(z)=P(z) \sum_{k, l} \frac{(-1)^{(k+l)} \alpha_{k, l}}{z-w_{k, l}} .
$$

In general the above sum does not even converge. The following lemma shows that under the additional assumptions on the double sequence $\alpha_{k, l}$ the series given by (5) converges uniformly on compact sets in $S$ and that the corresponding sum $\phi(z)$ is an integrable meromorphic function.

Lemma 2.1. Suppose that $\left\{\alpha_{k, l}\right\}, k, l \in \mathbf{Z}$, is a double sequence which satisfies the following properties:

$1^{o} \alpha_{k, l}=\alpha_{-l-1, k}=\alpha_{-k-1,-l-1}=\alpha_{l,-k-1}$.

$2^{o}$

$$
\sum_{k, l}\left|\alpha_{k, l}\right|\left|w_{k, l}\right|<\infty
$$

Then the corresponding function $\phi$ given by (5) is well defined and $\phi \in A^{1}$. Also $\phi\left(w_{k, l}\right)=\alpha_{k, l}$. 
Proof. By using the assumption $1^{o}$ we obtain the following identity:

$$
\begin{gathered}
\frac{(-1)^{(k+l)} \alpha_{k, l}}{z-w_{k, l}}+\frac{(-1)^{(-l-1+k)} \alpha_{-l-1, k}}{z-i w_{k, l}}+\frac{(-1)^{(-k-1-l-1)} \alpha_{-k-1,-l-1}}{z+w_{k, l}} \\
+\frac{(-1)^{(l-k-1)} \alpha_{l,-k-1}}{z+i w_{k, l}}=\frac{4(-1)^{(k+l)}\left(\alpha_{k, l} w_{k, l}^{2}\right) z}{z^{4}-w_{k, l}^{4}} .
\end{gathered}
$$

By combining the identities (6) and (5) we can rewrite the series given by (5) (without changing its sum) as

$$
\phi(z)=P(z) \sum_{k, l \geq 0} \frac{4(-1)^{(k+l)} \alpha_{k, l} w_{k, l}^{2} z}{z^{4}-w_{k, l}^{4}} .
$$

We show that the series given by (7) absolutely converges. By the change of variables we obtain

$$
\int_{\mathbf{C}} \frac{\left|4 w_{k, l}^{2} z\right|}{\left|z^{4}-w_{k, l}^{4}\right|} d x d y=4\left|w_{k, l}\right| \int_{\mathbf{C}} \frac{|z|}{\left|z^{4}-1\right|} d x d y=4\left|w_{k, l}\right| I
$$

where $z=x+i y$ and the last integral $I$ in (8) exists. Estimating the series given by (7) we have

$$
|\phi(z)| \leq|P(z)| \sum_{k, l \geq 0} \frac{4\left|\alpha_{k, l}\right|\left|w_{k, l}^{2}\right||z|}{\left|z^{4}-w_{k, l}^{4}\right|}
$$

for each $z \in S$. By using the identity (8) we obtain

$$
\int_{\mathbf{C}}\left(\sum_{k, l \geq 0} \frac{4\left|\alpha_{k, l}\right|\left|w_{k, l}^{2}\right||z|}{\left|z^{4}-w_{k, l}^{4}\right|}\right) d x d y=I \sum_{k, l \geq 0}\left|\alpha_{k, l}\right|\left|w_{k, l}\right| .
$$

From the assumption $2^{\circ}$ we conclude that the series given by (7) absolutely converges for each $z \in S$, and therefore $\phi(z)$ is a well defined analytic function on $S$. Moreover from the definition of $\phi$ it follows that $\phi$ has at most the first order poles at the points $z_{k, l} \in Z$.

To conclude that $\phi$ is integrable on $\mathbf{C}$ we start with the following estimate which is a consequence of the above two inequalities:

$$
\int_{\mathbf{S}_{1}}|\phi|<C_{1} I \sum_{k, l \geq 0}\left|\alpha_{k, l}\right|\left|w_{k, l}\right|
$$

where $C_{1}$ is the constant defined by $(2)$ and $\left(2^{\prime}\right)$. So we have that $\phi(z)$ is integrable on $S_{1}$. Since $\phi(z)$ has at most the first order poles at the points $z_{k, l}$, for each $z_{k, l}$ we obtain the following representation of the function $\phi(z)$ :

$$
\phi(z)=\frac{a_{k, l}(z)}{z-z_{k, l}}, \quad\left|z-z_{k, l}\right|<\frac{1}{2},
$$

where $a_{k, l}(z)$ are analytic functions in $\left|z-z_{k, l}\right|<\frac{1}{2}$ for each $(k, l)$. By using the above representation of the function $\phi(z)$ and by switching to the polar coordinates one concludes that the estimate

$$
\int_{\left|z-z_{k, l}\right| \leq \frac{1}{8}}|\phi|<\frac{1}{8\left(\frac{1}{2}-\frac{1}{8}\right)}\left(\int_{\frac{1}{8}<\left|z-z_{k, l}\right|<\frac{1}{2}}|\phi|\right)
$$


holds for each $z_{k, l}$. This follows from the fact that the integral means of the functions $\left|a_{k, l}(z)\right|$ increase with the increasing radius. Now by using (9) and (10) we find

$$
\int_{\mathbf{C}}|\phi|<2 C_{1} I \sum_{k, l \geq 0}\left|\alpha_{k, l}\right|\left|w_{k, l}\right|
$$

and therefore we have proved that $\phi \in A^{1}$. As we already have shown the fact that $\phi\left(w_{k, l}\right)=\alpha_{k, l}$ follows at once from the definition of $\phi$ and the properties of the function $P(z)$.

\section{Construction of the Sequence and main lemmas}

The objective of this section is to construct the sequence $\phi_{n}(z)$ which satisfies the assumptions of Proposition 1.2. We establish some important properties of this sequence and in this section we actually prove that $\phi_{n}(z)$ satisfies the conditions $1^{\circ}$ and $2^{\circ}$ of the Proposition 1.2. In order to construct this sequence we use the representation we introduced in the previous section, that is, we apply Lemma 2.1.

For each $n \in \mathbf{N}$ we define the double sequence $\left\{\alpha_{k, l}(n)\right\}$ by

$$
\alpha_{k, l}(n)=\frac{1}{\left(\frac{\left|w_{k, l}\right|}{n}+1\right)^{4}}, \quad n \in \mathbf{N} .
$$

It can be directly verified that the sequence $\alpha_{k, l}(n)$ satisfies the assumptions of Lemma 2.1 for each fixed $n \in \mathbf{N}$. Therefore by the same lemma we conclude that the function $\tilde{\phi}_{n}(z)$, given by

$$
\tilde{\phi}_{n}(z)=P(z) \sum_{k, l \in \mathbf{Z}} \frac{(-1)^{k+l} \alpha_{k, l}(n)}{z-w_{k, l}},
$$

is well defined. By applying the same lemma we have $\tilde{\phi}_{n} \in A^{1}$ and

$$
\tilde{\phi}_{n}\left(w_{k, l}\right)=\alpha_{k, l}(n) .
$$

Next, for each function $\varphi(z)$ defined in the complex plane we define the new function $\Theta(\varphi)$ by

$$
\Theta(\varphi)(z)=\frac{\varphi(z)+\varphi(z+1)}{2} .
$$

If $\varphi \in A^{1}$ we have $\Theta(\varphi) \in A^{1}$. We define the sequence of the function $\phi_{n}(z)$ as the eleventh iteration of the operator $\Theta$ evaluated in $\tilde{\phi}_{n}(z)$

$$
\phi_{n}(z)=\Theta^{(11)}\left(\tilde{\phi}_{n}\right)(z) \text {. }
$$

We have that $\phi_{n}(z)$ is an element of $A^{1}$ for each $n \in \mathbf{N}$. Also one can express the function $\phi_{n}(z)$ as the fixed linear combination (independent of $n$ ) of the functions $\tilde{\phi}_{n}(z), \tilde{\phi}_{n}(z+1), \ldots, \tilde{\phi}_{n}(z+11)$. In this section we show that the sequence given by $\left(12^{\prime}\right)$ satisfies the first two assumptions of Proposition 1.2.

Remark. We remark that in the definition (11) of the double sequence $\alpha_{k, l}(n)$ we could have taken that the exponent is an arbitrary integer $\geq 4$. Then by taking the larger order of the corresponding iteration of $\Theta$ we would obtain other examples of sequences satisfying the assumptions of Proposition 1.2. The choice of this exponent and the choice of the eleventh iteration in the definition $\left(12^{\prime}\right)$ are the technical details and the meaning of this particular choice will be understood from 
the proof of Lemma 3.2. Although it is irrelevant we remark that one can choose the lower order of the iteration in the definition $\left(12^{\prime}\right)$ (but not lower than 8).

In the remainder of this paper the notation $\phi_{n}(z), \tilde{\phi}_{n}(z), \alpha_{k, l}(n)$ is reserved for the above defined objects. First we prove the following lemma which gives the needed information about the sequence $\left\{\alpha_{k, l}(n)\right\}$.

Lemma 3.1. Let $(p, q)$ be a fixed pair of integers. Then the sequence $\left\{\alpha_{k, l}(n)\right\}$ given by (11) satisfies the following inequality:

$$
\left|n \alpha_{k, l}(n)\left(\frac{\left|w_{p, q}\right|}{n}+1\right)^{5}-n\left(\frac{\left|w_{p, q}\right|}{n}+1\right)\right| \leq 4\left|w_{k, l}-w_{p, q}\right|\left(1+\left|w_{k, l}-w_{p, q}\right|\right)^{4}
$$

for each pair $(k, l)$ and for each $n \in \mathbf{N}$.

Proof. For each $n \in \mathbf{N}$ we define the function $f_{n}(x, y)$ by

$$
f_{n}(x, y)=n \frac{\left(\frac{x}{n}+1\right)}{\left(\frac{y}{n}+1\right)^{4}}\left|\left(\frac{x}{n}+1\right)^{4}-\left(\frac{y}{n}+1\right)^{4}\right|
$$

for $x, y>0$. By applying the Lagrange mean value theorem onto the function $\left(\frac{t}{n}+1\right)^{4}$ where $t>0$ we can estimate the function $f_{n}(x, y)$ in the following way:

$$
f_{n}(x, y) \leq n \frac{\left(\frac{x}{n}+1\right)}{\left(\frac{y}{n}+1\right)^{4}}\left|\frac{x}{n}-\frac{y}{n}\right| 4 \max \left\{\left(\frac{x}{n}+1\right)^{3},\left(\frac{y}{n}+1\right)^{3}\right\} .
$$

Now we have two cases. First if $y \geq x$ we obtain

$$
f_{n}(x, y) \leq 4|x-y|
$$

directly from (15). If $x>y$ we have

$$
\frac{\left(\frac{x}{n}+1\right)^{4}}{\left(\frac{y}{n}+1\right)^{4}} \leq \frac{(x+1)^{4}}{(y+1)^{4}} \leq(1+|x-y|)^{4} .
$$

By combining the above inequality with (15) we obtain

$$
f_{n}(x, y) \leq 4|x-y|(1+|x-y|)^{4}, \quad x>y .
$$

Together (15) and (17) imply that the following estimate holds for each $x, y>0$ and each $n \in \mathbf{N}$ :

$$
f_{n}(x, y) \leq 4|x-y|(1+|x-y|)^{4} .
$$

Now we take $\left|w_{p, q}\right|=x$ and $\left|w_{k, l}\right|=y$. With this substitution the expression $f_{n}(x, y)$ defined by (14) agrees with the left side of the inequality (13). Since ||$w_{k, l}|-| w_{p, q}|| \leq\left|w_{k, l}-w_{p, q}\right|$ the estimate (13) follows from the corresponding estimate (18) for the function $f_{n}(x, y)$.

In the next lemma we derive all necessary information about the sequence $\phi_{n}$. All further results related to the sequence $\phi_{n}(z)$ given by $\left(12^{\prime}\right)$ are the consequences of Lemma 3.2. 
Lemma 3.2. Let $w_{p, q}$ be an arbitrary fixed element of $W$. Let $\boldsymbol{\Delta}_{p, q}=\left\{\left|z-w_{p, q}\right|<\right.$ $1\}$. Then there exists an absolute constant $C_{2}>0$, independent of the choice of $n \in \mathbf{N}$ or the pair $(p, q)$, such that the estimate

$$
\left|n \phi_{n}(z)\left(\frac{\left|w_{p, q}\right|}{n}+1\right)^{4}-n\right| \leq C_{2}
$$

holds for $z \in \boldsymbol{\Delta}_{p, q} \cap S_{1}$. Moreover for each $z \in S_{1} \cap \boldsymbol{\Delta}_{p, q}$ we have

$$
\left|\phi_{n}(z)\right|-\operatorname{Re}\left(\phi_{n}(z)\right) \leq \frac{C_{2}^{2}}{n^{2}\left(1-\frac{C_{2}}{n}\right)\left(\frac{\left|w_{p, q}\right|}{n}+1\right)^{4}}
$$

where the constant $C_{1}$ is defined by the relation (2) in the previous section.

Proof. We first prove the inequality (19). The inequality $\left(19^{\prime}\right)$ is a corollary of (19) as we will see. Set

$$
R_{n}(z)=\sum_{k, l} \frac{(-1)^{(k+l)} \alpha_{k, l}(n)}{z-w_{k, l}}
$$

We note that the sequence $\tilde{\phi}_{n}(z)$ is given as $\tilde{\phi}_{n}(z)=P(z) R_{n}(z)$. Also set $P_{1}(z)=$ $\frac{1}{P(z)}$.

Next let $w_{p, q}$ be an arbitrary fixed element of the lattice $W$ (we save the notation $w_{k, l}$ for all other elements of $\left.W\right)$. Define the function $\psi_{p, q}(n)(z)$ by

$$
\left(n R_{n}(z)\left(\frac{\left|w_{p, q}\right|}{n}+1\right)^{4}-n P_{1}(z)\right)=\psi_{p, q}(n)(z) .
$$

From the definition of $\psi_{p, q}(n)(z)$ we find that $\psi_{p, q}(n)(z)$ is an analytic function in $\mathbf{C} \backslash W$ and it has the first order poles at the points $w_{k, l}$ whose residues $\lambda_{k, l}(n)$ are given by

$$
\lambda_{k, l}(n)=(-1)^{k+l}\left[n \alpha_{k, l}(n)\left(\frac{\left|w_{p, q}\right|}{n}+1\right)^{4}-n\right] .
$$

We have $\lambda_{p, q}(n)=0$ so the function $\psi_{p, q}(n)(z)$ extends analytically over the point $w_{p, q}$. Consider the Taylor expansion of the function $\psi_{p, q}(n)(z)$ centered at the point $w_{p, q}$ and let $T_{p, q}(n)(z)$ be the polynomial of the degree 10 such that $\psi_{p, q}(n)(z)$ satisfies the relation

$$
\psi_{p, q}(n)(z)=T_{p, q}(n)(z)+O\left(\left(z-w_{p, q}\right)^{11}\right)
$$

for $z$ close to $w_{p, q}$. Now we define the new function $\varphi_{p, q}(n)(z)$ by

$$
\varphi_{p, q}(n)(z)=\frac{\psi_{p, q}(n)(z)-T_{p, q}(n)(z)}{\left(z-w_{p, q}\right)^{11}} .
$$

The function $\varphi_{p, q}(n)(z)$ is a meromorphic function and it has the first order poles at the points $w_{k, l} \neq w_{p, q}$. In particular $\varphi_{p, q}(n)(z)$ is analytic at the point $w_{p, q}$. The residues $\gamma_{k, l}(n)$ of $\varphi_{p, q}(n)(z)$ at the points $w_{k, l} \neq w_{p, q}$ are given by

$$
\gamma_{k, l}(n)=\frac{\lambda_{k, l}(n)}{\left(w_{k, l}-w_{p, q}\right)^{11}} .
$$


By the application of Lemma 3.1 we obtain the estimate

$$
\left|\gamma_{k, l}(n)\right| \leq 4 \frac{\left|w_{k, l}-w_{p, q}\right|\left(1+\left|w_{k, l}-w_{p, q}\right|\right)^{4}}{\left|w_{k, l}-w_{p, q}\right|^{11}\left(\frac{\left|w_{p, q}\right|}{n}+1\right)} \leq 4 \frac{\left|w_{k, l}-w_{p, q}\right|\left(1+\left|w_{k, l}-w_{p, q}\right|\right)^{4}}{\left|w_{k, l}-w_{p, q}\right|^{11}}
$$

for each pair $(k, l) \neq(p, q)$. Define the function $D_{p, q}(n)(z)$ by

$$
D_{p, q}(n)(z)=\sum_{(k, l) \neq(p, q)} \frac{\gamma_{k, l}(n)}{z-w_{k, l}}
$$

We will soon see that $D_{p, q}(n)(z)$ is well defined and that $D_{p, q}(n)(z)=\varphi_{p, q}(n)(z)$.

First we estimate $D_{p, q}(n)(z)$. Since $\gamma_{p, q}(n)=0$ we simply estimate $\left|D_{p, q}(n)(z)\right|$ as

$$
\left|D_{p, q}(n)(z)\right| \leq \sum_{(k, l) \neq(p, q)} \frac{\left|\gamma_{k, l}(n)\right|}{\left|z-w_{k, l}\right|}
$$

By applying the estimate (13) from Lemma 3.1 we obtain the following chain of inequalities:

$$
\begin{gathered}
\left|D_{p, q}(n)(z)\right| \leq \sum_{(k, l) \neq(p, q)} \frac{4 \frac{\left|w_{k, l}-w_{p, q}\right|\left(1+\left|w_{k, l}-w_{p, q}\right|\right)^{4}}{\left|w_{k, l}-w_{p, q}\right|^{11}}}{\left|z-w_{k, l}\right|} \\
=\sum_{(k, l) \neq(0,0)} 4 \frac{|k+i l|(1+|k+i l|)^{4}}{|k+i l|^{11}\left|z-w_{p+k, q+l}\right|} .
\end{gathered}
$$

It is clear that the last sum in (22) exists for each $z \in \mathbf{C} \backslash W$ and that it does not depend on $n \in \mathbf{N}$.

In order to show $D_{p, q}(n)(z)=\varphi_{p, q}(n)(z)$ we consider arbitrary sequence of complex numbers $\left\{z_{m}\right\}, m \in \mathbf{N}$, such that $z_{m} \rightarrow \infty, m \rightarrow \infty$ and such that the distance between $z_{m}$ and $W$ is positive and uniformly bounded from below for all $m \in \mathbf{N}$. Then directly from $(22)$ it follows that $D_{p, q}(n)\left(z_{m}\right) \rightarrow 0$. The same fact holds for the function $\varphi_{p, q}(n)(z)$. Moreover we have $\varphi_{p, q}(n)\left(z_{m}\right)=O\left(\frac{1}{\left|z_{m}\right|^{11}}\right)$ for fixed $n \in \mathbf{N}$ and pair $(p, q)$ (see the definition of $\varphi_{p, q}(n)(z)$ ). Functions $D_{p, q}(n)(z)$ and $\varphi_{p, q}(n)(z)$ have the same singular parts at the points $w_{k, l}$ and we conclude that $D_{p, q}(n)(z)-\varphi_{p, q}(n)(z)$ is a bounded entire function approaching zero when $z$ approaches $\infty$. Therefore $D_{p, q}(n)(z)=\varphi_{p, q}(n)(z)$.

So we have

$$
\left|\varphi_{p, q}(n)(z)\right| \leq \sum_{(k, l) \neq(0,0)} 4 \frac{|k+i l|(1+|k+i l|)^{4}}{|k+i l|^{11}\left|z-w_{p+k, q+l}\right|}
$$

for each pair $(p, q)$ and $z \in \mathbf{C} \backslash W$. If we multiply both sides in the above inequality by $|P(z)|$ we obtain

$$
\left|P(z) \varphi_{p, q}(n)(z)\right| \leq|P(z)| \sum_{(k, l) \neq(0,0)} 4 \frac{|k+i l|(1+|k+i l|)^{4}}{|k+i l|^{11}\left|z-w_{p+k, q+l}\right|}
$$

for each pair $(p, q)$ and $z \in \mathbf{C} \backslash W$. But from the above estimate we see that the function $P(z) \varphi_{p, q}(n)(z)$ is uniformly bounded on the whole domain $S_{1}$. Indeed, 
from $\left(2^{\prime}\right)$ we have

$$
\frac{|P(z)|}{\left|z-w_{k, l}\right|} \leq C_{1}
$$

for each pair $(k, l)$ and $z \in S_{1}$. We conclude that the estimate

$$
\left|P(z) \varphi_{p, q}(n)(z)\right| \leq C_{1} \sum_{(k, l) \neq(0,0)} 4 \frac{|k+i l|(1+|k+i l|)^{4}}{|k+i l|^{11}}=C_{1} C_{2}^{\prime}
$$

holds for each $z \in S_{1}$, and the constant $C_{2}^{\prime}$ represents the sum of the above series. Now we apply definition (12) and the definition of $\psi_{p, q}(n)$. By multiplying the above inequality by $\left|z-w_{p, q}\right|^{11}$ we obtain that the inequality

$$
\begin{aligned}
|P(z)|\left|\psi_{p, q}(n)(z)-T_{p, q}(n)(z)\right| & =\left|n \tilde{\phi}_{n}(z)\left(\frac{\left|w_{p, q}\right|}{n}+1\right)^{4}-n-P(z) T_{p, q}(n)(z)\right| \\
\leq & C_{1} C_{2}^{\prime}\left|z-w_{p, q}\right|^{11}
\end{aligned}
$$

holds for each $n \in \mathbf{N}$, pair $(p, q)$ and every $z \in S_{1}$. In particular we choose the constant $C_{2}$ such that the inequality

$$
\left|n \tilde{\phi}_{n}(z)\left(\frac{\left|w_{p, q}\right|}{n}+1\right)^{4}-n-P(z) T_{p, q}(n)(z)\right| \leq C_{2}
$$

holds for each $z \in S_{1}$ which satisfies the condition $\left|z-w_{p, q}\right| \leq 20$. We now apply the operator $\Theta$ to the function whose absolute value is on the left side of the inequality (23). But first note that if $T(z)$ is an arbitrary polynomial of some degree $m \in \mathbf{N}$ we have that the degree of the polynomial $T(z+1)-T(z)$ is equal to $m-1$. After one application of $\Theta$ from (23) we obtain

$$
\begin{aligned}
\mid n\left(\frac{\tilde{\phi}_{n}(z+1)+\tilde{\phi}_{n}(z)}{2}\right) & \left(\frac{\left|w_{p, q}\right|}{n}+1\right)^{4} \\
& -n-P(z)\left(\frac{T_{p, q}(n)(z)-T_{p, q}(n)(z+1)}{2}\right) \mid \leq C_{2} .
\end{aligned}
$$

The above estimate holds for $z \in S_{1}$ and $\left|z-w_{p, q}\right| \leq 19$. In the expression on the left side of (24) we have used the fact that $P(z)=-P(z+1)$. We now repeat the process 10 times. We take the eleventh iteration of the expression whose absolute value is on the left side of inequality (23). The resulting inequality of this process is inequality (19) in the statement of this lemma and it holds for each $z \in S_{1}$ and $\left|z-w_{p, q}\right| \leq 9$ (the corresponding polynomial is of the degree zero in the eleventh iteration). In particular we have that inequality (19) holds for $z \in S_{1} \cap \boldsymbol{\Delta}_{p, q}$.

Suppose that $K>0$ and $w \in \mathbf{C}$ are two arbitrary numbers such that $K>|w|$. We have the estimate

$$
|K+w|-\operatorname{Re}(K+w) \leq \frac{\operatorname{Im}^{2}(w)}{K-|w|}
$$

We set $K=n$ and

$$
w=n \phi_{n}(z)\left(\frac{\left|w_{p, q}\right|}{n}+1\right)^{4}-n .
$$

With this substitution and by combining inequalities (19) and (25) we derive inequality $\left(19^{\prime}\right)$. 
The previous lemma gives an estimate good enough for the expression $\left|\phi_{n}(z)\right|-$ $\operatorname{Re}\left(\phi_{n}(z)\right)$ in the case $z \in S_{1}$. And we will soon see that this estimate directly implies that the sequence

$$
\int_{S_{1}}\left|\phi_{n}\right|-\operatorname{Re}\left(\phi_{n}\right)
$$

is uniformly bounded. But if $z$ is not in $S_{1}$, then the estimates (19) and (19') do not hold anymore for such $z$ because one can find the point $z \in S \backslash S_{1}$, which is very close to the point $z_{k, l}$, which is a possible pole of $\phi_{n}(z)$. However it is natural to expect that just by using inequality (19) (which holds on $S_{1}$ ) one can prove that there exists the corresponding integral form of inequality $\left(19^{\prime}\right)$ in the neighborhood of the point $z_{k, l}$ for each $(k, l)$. That would result in the way that the sequence of integrals of the functions $\left|\phi_{n}\right|-R e\left(\phi_{n}\right)$ is uniformly bounded if the domain of integration is the whole complex plane. This is exactly the second assumption of Proposition 1.2. The following proposition resolves this issue.

Proposition 3.1. Suppose that $a(z)$ is an analytic function on the unit disc which satisfies

$$
|a(z)|<C_{3}
$$

for $|z|<\frac{1}{2}$. Let $K$ be an arbitrary positive number which satisfies the condition $K>8 C_{3}$. Then there exists a constant $C_{4}$ which depends only on the upper bound $C_{3}$ such that the estimate

$$
\int_{|z|<\frac{1}{8}}\left(\left|\frac{a(z)}{z}+K\right|-\operatorname{Re}\left(\frac{a(z)}{z}+K\right)\right) d x d y \leq \frac{C_{4}}{K}
$$

holds.

Proof. We apply the Green's formula to the functions $|z|^{2}$ and $u(z)=\left|\frac{a(z)}{z}+K\right|-$ $\operatorname{Re}\left(\frac{a(z)}{z}+K\right)$ over the domain $\boldsymbol{\Delta}_{r}=\left\{r<|z|<\frac{1}{8}\right\}$. Although the function $u(z)$ is not of the class $C^{2}$ at every point of the domain $\boldsymbol{\Delta}_{r}$ the Green's formula remains valid in this case (see the remark in the next section). We have

$$
\begin{gathered}
\int_{\boldsymbol{\Delta}_{r}}\left(4 u(z)-|z|^{2} \Delta u(z)\right) d x d y=\int_{|z|=\frac{1}{8}}\left(\frac{\partial|z|^{2}}{\partial \mathbf{n} u(z)-\frac{\partial u(z)}{\partial \mathbf{n}}|z|^{2}}\right)|d z| \\
+\int_{|z|=r}\left(\frac{\partial|z|^{2}}{\partial \mathbf{n}} u(z)-\frac{\partial u(z)}{\partial \mathbf{n}}|z|^{2}\right)|d z| .
\end{gathered}
$$

Here $\frac{\partial}{\partial \mathbf{n}}$ denotes the derivative taken with the respect to the outer normal vector $n$. It is clear that the last integral on the right side of the above formula tends to zero when $r \rightarrow 0$. So by letting $r \rightarrow 0$ and since $u(z)$ is a positive subharmonic functions we obtain

$$
4 \int_{|z|<\frac{1}{8}} u(z) d x d y \leq \int_{|z|<\frac{1}{8}}|z|^{2} \Delta u(z) d x d y+\int_{|z|=\frac{1}{8}}\left(\left|\frac{\partial|z|^{2}}{\partial \mathbf{n}} u(z)\right|+\left.\left|\frac{\partial u(z)}{\partial \mathbf{n}}\right| z\right|^{2} \mid\right)|d z| .
$$


Set

$$
v(z)=K+\frac{a(z)}{z} .
$$

We have the the following equality:

$$
\frac{\partial u(z)}{\partial \mathbf{n}}=\frac{\frac{\partial \operatorname{Re}(v(z))}{\partial \mathbf{n}} \operatorname{Re}(v(z))+\frac{\partial \operatorname{Im}(v(z))}{\partial \mathbf{n}} \operatorname{Im}(v(z))}{|v(z)|}-\frac{\partial \operatorname{Re}(v(z))}{\partial \mathbf{n}} .
$$

For $|z|=\frac{1}{8}$ we obtain the estimates

$$
\begin{gathered}
\left|\frac{\frac{\partial \operatorname{Im}(v(z))}{\partial \mathbf{n}} \operatorname{Im}(v(z))}{|v(z)|}\right| \leq \frac{8 C_{3} C_{3}^{\prime}}{K-8 C_{3}}, \\
\left|\frac{\frac{\partial \operatorname{Re}(v(z))}{\partial \mathbf{n}} \operatorname{Re}(v(z))}{|v(z)|}-\frac{\partial \operatorname{Re}(v(z))}{\partial \mathbf{n}}\right| \leq \frac{\left(8 C_{3}\right)^{2} C_{3}^{\prime}}{\left(K-8 C_{3}^{\prime}\right)^{2}} .
\end{gathered}
$$

Together the above estimates yield

$$
\left|\frac{\partial u(z)}{\partial \mathbf{n}}\right| \leq \frac{8 C_{3} C_{3}^{\prime}}{K-8 C_{3}}+\frac{\left(8 C_{3}\right)^{2} C_{3}^{\prime}}{\left(K-8 C_{3}\right)^{2}}=O\left(\frac{1}{K}\right)
$$

where $C_{3}^{\prime}$ is the upper bound of the absolute value of the first derivative $\left(\frac{a(z)}{z}\right)^{\prime}$ for $|z|=\frac{1}{8}$. We have that $C_{3}^{\prime}$ depends only on $C_{3}$. From the above estimate we derive the relation

$$
\left.\int_{|z|=\frac{1}{8}}\left|\frac{\partial u(z)}{\partial \mathbf{n}}\right| z\right|^{2}|| d z \mid=O\left(\frac{1}{K}\right) .
$$

Similarly by applying the estimate (25) we obtain

$$
\int_{|z|=\frac{1}{8}}\left|\frac{\partial|z|^{2}}{\partial \mathbf{n}} u(z)\right||d z| \leq \frac{\left(8 C_{3}\right)^{2}}{K-8 C_{3}}=O\left(\frac{1}{K}\right) .
$$

It remains to estimate the first integral on the right side of the inequality (26). First one verifies that the formula

$$
\Delta u(z)=\frac{\left|v(z)^{\prime}\right|^{2}}{|v(z)|}
$$

holds for each $z \neq 0$ (see the following section). By applying the above equality we have

$$
I_{1}=\int_{\frac{2 C_{3}}{K}<|z|<\frac{1}{8}}|z|^{2} \Delta u(z) d x d y \leq \int_{\frac{2 C_{3}}{K}<|z|<\frac{1}{8}} \frac{\left(C_{3}^{\prime}\right)^{2}}{K-\frac{K}{2}} d x d y \leq \frac{2 \pi\left(C_{3}^{\prime}\right)^{2}}{K} .
$$

Next, by the change of variables and from substitution $z=\frac{2 C_{3} w}{K}$ we obtain

$$
I_{2}=\int_{|z|<\frac{2 C_{3}}{K}}|z|^{2} \Delta u(z) d x d y=\frac{\left(2 C_{3}\right)^{2}}{K} \int_{|w|<1}|w|^{2} \Delta\left|\frac{a\left(\frac{2 C_{3}}{K} w\right)}{2 C_{3} w}+1\right| d w_{1} d w_{2}
$$


where $w=w_{1}+i w_{2}$. The integral

$$
\int_{|w|<1}|w|^{2} \Delta\left|\frac{a\left(\frac{2 C_{3}}{K} w\right)}{2 C_{3} w}+1\right| d w_{1} d w_{2}
$$

is uniformly bounded and that bound depends only on $C_{3}$. That can be verified directly by applying the Green's theorem to the functions $|w|^{2}$ and $b(w)=$ $\left|\frac{a\left(\frac{2 C_{3}}{K} w\right)}{2 C_{3} w}+1\right|$. By estimating the resulting relation in the same way as we did above we obtain the following inequality (similar to the inequality (26)):

$$
\begin{gathered}
\int_{|w|<1}|w|^{2} \Delta b(w) d w_{1} d w_{2} \leq \int_{|w|<1} 4 b(w) d w_{1} d w_{2} \\
+\int_{|w|=1}\left(\left|\frac{\partial|w|^{2}}{\partial \mathbf{n}} b(w)\right|+\left.\left|\frac{\partial b(w)}{\partial \mathbf{n}}\right| w\right|^{2} \mid\right)|d w|=O\left(C_{3}\right) .
\end{gathered}
$$

The previous conclusion is also a corollary of Proposition 4.1 from the next section.

From the previous inequalities we see that both $I_{1}$ and $I_{2}$ are of the form $O\left(\frac{1}{K}\right)$ whenever the upper bound $C_{3}$ is fixed. Since $I=I_{1}+I_{2}$ we conclude that there exists a constant $C_{4}$ such that the proposition holds.

We are now ready to prove that our sequence $\phi_{n}(z)$ satisfies the first two properties of Proposition 1.2. We have

Lemma 3.3. The sequence $\phi_{n}(z)$ given by $\left(12^{\prime}\right)$ satisfies the conditions:

$1^{o} \lim _{n \rightarrow \infty} \phi_{n}(z)=1$, for $z \in S$.

$2^{o}$ There exists a constant $C$ such that

$$
\int_{\mathbf{C}}\left|\phi_{n}\right|-\operatorname{Re}\left(\phi_{n}\right) \leq C \text {. }
$$

Proof. We first show that condition $2^{o}$ is satisfied. Set $E_{p, q}=\boldsymbol{\Delta}_{p, q} \cap S_{1}$. From the inequality $\left(19^{\prime}\right)$ we conclude

$$
\int_{E_{p, q}}\left(\left|\phi_{n}(z)\right|-\operatorname{Re}\left(\phi_{n}(z)\right)\right) d x d y \leq \frac{\pi C_{2}^{2}}{n^{2}\left(1-\frac{C_{2}}{n}\right)\left(\frac{\left|w_{p, q}\right|}{n}+1\right)^{4}} .
$$

Next we note that the set $\Delta p, q-E_{p, q}$ is a union of four discs centered at the points $z_{p, q}, z_{p+1, q}, z_{p, q+1}, z_{p+1, q+1}$ of radii $\frac{1}{8}$. We set the substitutions $K=n$ and for $i, j=0,1$ we set

$$
\frac{a_{i, j}(z)}{z}=n \phi_{n}\left(z+z_{p+i, q+j}\right)\left(\frac{\left|w_{p, q}\right|}{n}+1\right)^{4}-n .
$$

From the inequality (19) we conclude that the inequality $\frac{\left|a_{i, j}(z)\right|}{|z|} \leq C_{2}$, holds for $\frac{1}{8} \leq$ $\left|z-z_{k+i, l+j}\right| \leq \frac{1}{2}$. Therefore we can apply Proposition 3.1 onto the corresponding $K$ (for $n$ large enough we have that the corresponding $K$ satisfies the assumption of Proposition 3.1) and four times respectively to each $a_{i, j}(z)$. We obtain

$$
\sum_{i, j=0}^{1} \int_{|z|<\frac{1}{8}}\left|\phi_{n}\right|-\operatorname{Re}\left(\phi_{n}\right) \leq \frac{4 C_{4}}{n^{2}\left(\frac{\left|w_{p, q}\right|}{n}+1\right)^{4}} .
$$


By combining the above estimate and the estimate (27) we derive the following:

$$
\begin{gathered}
\int_{\frac{\Delta_{p, q}}{}\left|\phi_{n}(z)\right|-} \operatorname{Re}\left(\phi_{n}(z)\right) d x d y \leq \frac{\pi C_{2}^{2}}{n^{2}\left(1-\frac{C_{2}}{n}\right)\left(\frac{\left|w_{p, q}\right|}{n}+1\right)^{4}}+4 C_{4} \\
n^{2}\left(\frac{\left|w_{p, q}\right|}{n}+1\right)^{4} \\
\leq \frac{2 \max \left\{\pi C_{2}^{2}, 4 C_{4}\right\}}{n^{2}\left(1-\frac{C_{2}}{n}\right)\left(\frac{\left|w_{p, q}\right|}{n}+1\right)^{4}} .
\end{gathered}
$$

Since the family of discs $\boldsymbol{\Delta}_{p, q}$ covers the whole plain, after the summation over all $p, q \in \mathbf{Z}$ we obtain

$$
\int_{\mathbf{C}}\left|\phi_{n}\right|-\operatorname{Re}\left(\phi_{n}(z)\right) d x d y \leq \sum_{p, q} \frac{2 \max \left\{\pi C_{2}^{2}, 4 C_{4}\right\}}{n^{2}\left(1-\frac{C_{2}}{n}\right)\left(\frac{\left|w_{p, q}\right|}{n}+1\right)^{4}} .
$$

The series on the right side of the previous inequality can be estimated as

$$
\sum_{p, q} \frac{1}{n^{2}\left(\frac{\left|w_{p, q}\right|}{n}+1\right)^{4}} \leq \int_{\mathbf{C}} \frac{d x d y}{n^{2}\left(\frac{|z|}{n}+1\right)^{4}}+o(1)=I_{n}+o(1)
$$

where $o(1) \rightarrow 0$ when $n \rightarrow \infty$. Moreover by switching to the polar coordinates we evaluate the integral $I_{n}$ as

$$
I_{n}=\int_{0}^{\infty} \frac{r d r}{n^{2}\left(\frac{r}{n}+1\right)^{4}}=\int_{0}^{\infty} \frac{t d t}{(t+1)^{4}}=I .
$$

From (28) we find

$$
\int_{\mathbf{C}}\left|\phi_{n}\right|-R e\left(\phi_{n}\right) \leq I \frac{\max \left\{\pi C_{2}^{2}, 4 C_{4}\right\}}{1-\frac{C_{2}}{n}}+o(1) .
$$

Now it is clear that we can choose a constant $C$ so that the condition $2^{\circ}$ of this lemma is satisfied.

Next we prove that the sequence $\phi_{n}(z)$ satisfies the condition $1^{\circ}$. We know that $\tilde{\phi}_{n}\left(w_{p, q}\right)=\alpha_{p, q}(n) \rightarrow 1$ when $n \rightarrow \infty$ for each fixed $w_{p, q}$. Because of that and from the definition $\left(12^{\prime}\right)$ we conclude that $\phi_{n}\left(w_{p, q}\right) \rightarrow 1$ when $n \rightarrow \infty$ for each fixed $w_{p, q}$. We just have proved that the sequence $\phi_{n}(z)$ satisfies the condition $2^{o}$ of this lemma. By applying the mean value property for analytic function we conclude that the sequence of integrals of $\left|\phi_{n}(z)\right|$ is bounded on every compact subset in $S$. Therefore the sequence $\phi_{n}(z)$ is a normal family. That means that any subsequence of $\phi_{n}(z)$ converges to some analytic function $\phi(z)$ on $S$. Moreover from inequality $\left(19^{\prime}\right)$ we conclude that for each $z \in S$ we have $\left|\phi_{n}(z)\right|-\operatorname{Re}\left(\phi_{n}(z)\right) \rightarrow 0$ and as a conclusion we have $\phi(z)=1, z \in S$ for any possible limit function $\phi(z)$. This proves that $\phi_{n}(z)$ satisfies the condition $1^{o}$.

\section{Proof of Theorem 1.1}

In this section we prove that the sequence $\phi_{n}(z)$ given by $\left(12^{\prime}\right)$ satisfies the third assumption of Proposition 1.2. The final conclusion is given by Lemma 4.3. First we investigate the meaning of the corresponding third condition from Proposition 1.2. The following results are related to the local and global properties of a certain wide class of integrable meromorphic functions. 
Lemma 4.1. Suppose that a $(z)$ is an analytic function in the disc $\boldsymbol{\Delta}_{1}$ of radius $\frac{1}{8}$ centered at the origin. In addition suppose that $\lambda$ is a complex number and let $K_{0}$ be any positive number such that the following holds:

$$
\left|a(z)+\frac{\lambda}{z}\right| \leq K_{0}
$$

for $|z|=\frac{1}{8}$. Then if $K>3 K_{0}$ and if $\Omega_{K}=\left\{z \in \boldsymbol{\Delta}_{1}:\left|a(z)+\frac{\lambda}{z}\right| \geq K\right\}$ we have the estimate

$$
\int_{\Omega_{K}}\left|a(z)+\frac{\lambda}{z}\right| d x d y \leq \frac{4 \pi \lambda^{2}}{K-2 K_{0}} .
$$

Proof. Note that from assumption (29) we obtain $|z a(z)+\lambda| \leq|z| K_{0}$ for $|z|=\frac{1}{8}$. It follows from the maximum principle that $|\lambda| \leq \frac{K_{0}}{8}$. By applying the triangle inequality and by using the fact that $a(z)$ is analytic we obtain $|a(z)| \leq 2 K_{0}$. If we consider the inequality

$$
\left|a(z)+\frac{\lambda}{z}\right| \geq K
$$

we conclude that $\Omega_{K}$ is contained in the disc $\Delta_{K}=\left\{|z| \leq \frac{\lambda}{K-2 K_{0}}\right\}$. Further we have

$$
\int_{\Omega_{K}}\left|a(z)+\frac{\lambda}{z}\right| d x d y \leq \int_{\Delta_{K}}\left|a(z)+\frac{\lambda}{z}\right| d x d y \leq \frac{2 \pi \lambda^{2} K_{0}}{\left(K-2 K_{0}\right)^{2}}+\frac{2 \pi \lambda^{2}}{\left(K-2 K_{0}\right)} .
$$

Since we have assumed that $K>3 K_{0}$ inequality (29) follows directly from the previous inequality.

It is clear from the previous lemma that we need to estimate the squares of residues of the functions $\phi_{n}(z)$. One can easily derive the satisfactory estimate directly from the considerations in Section 3. But we want to give the more general estimate of this type (which does not depend of the particular choice of the sequence $\left.\phi_{n}(z)\right)$ and thus we have the following.

First we recall a few basic facts about subharmonic functions (see [7]). Suppose that $U$ is a bounded domain in the complex plane and let $v(z)$ be a nonnegative, locally integrable subharmonic function given on $U$. Suppose that $C_{0}^{\infty}(U)$ denotes the space of all infinitely differentiable functions with compact support in $U$. Moreover suppose that function $v(z)$ has a weak Laplacian $\Delta v$ which is a locally finite Lebesgue measurable function, which satisfies the following Green's formula:

$$
\int_{U} \tau(\Delta v) d \mathbf{m}=\int_{U} v(\Delta \tau) d \mathbf{m},
$$

where $d \mathbf{m}$ is the derivative of the Lebesgue measure, and $\tau \in C_{0}^{\infty}(U)$.

Let $V$ denote a domain compactly contained in $U, \bar{V} \subset U$. The following statement is well known. For the sake of completeness, we include the short proof.

Proposition 4.1. With the notation stated above we have that there exists a constant $C(U, V)$, such that the following holds:

$$
\int_{V} \Delta v \leq C(U, V) \int_{U} v d \mathbf{m}
$$


where the constant $C(U, V)$ depends only on the choice of the domains $U$ and $V$ and not on the choice of the function $v(z)$.

Proof. Let us choose the function $\tau, \tau \in C_{0}^{\infty}(U)$, which satisfies

$$
\tau(z) \geq 0, \quad z \in U,
$$

and

$$
\tau(z)=1, \quad z \in V
$$

Put

$$
C(U, V)=\max _{z \in U}|\Delta \tau|(z) .
$$

Since $v(z)$ is nonnegative, by applying the Green's formula (31) we obtain

$$
\int_{V}(\Delta v) d \mathbf{m} \leq \int_{U} \tau(\Delta v) d \mathbf{m}=\int_{U} v(\Delta \tau) d \mathbf{m} \leq C(U, V) \int_{U} v d \mathbf{m} .
$$

From the last inequality we obtain that inequality (32) holds.

If $a(z)$ is an analytic function defined on the domain $U$, then $|a(z)|$ is a nonnegative subharmonic function on $U$. The weak Laplacian of $|a(z)|$ is the locally finite, Lebesgue measurable function given by

$$
\Delta|a|=\left|\frac{\left(a^{\prime}\right)^{2}}{a}\right|
$$

Remark. We note that the function $|a(z)|$ is not necessarily of the class $C^{2}$, so one cannot apply the classical Green's formula directly because it requests that the functions involved are of the class $C^{2}$. But we can apply the classical Green's formula onto the reduced domain of integration. That is if we take out the small discs surrounding the points where $|a(z)|$ is not of the class $C^{2}$ and by letting the radii of these discs to tend to zero, one would have that the corresponding singular parts tend to zero. That implies that the Green's formula remains valid in this case (see [7]). The Laplacian $\Delta|a(z)|$ is the absolute value of the locally integrable meromorphic function $\left|\frac{\left(a^{\prime}\right)^{2}}{a}\right|$ (it has at most the first order poles). So the Green's formula (31) holds if we apply it on the function $|a(z)|$.

We have the following corollary of Proposition 4.1.

Lemma 4.2. Let $\phi \in A^{1}$. Then there is a universal constant $C_{5}$ such that the following inequality holds:

$$
\int_{S_{1}} \Delta|\phi| \leq C_{5} \int_{\mathbf{C}}|\phi|-R e(\phi) .
$$

Proof. Set

$$
U=[-1<x<2]^{2}-\{0,1, i, 1+i\}, \quad V=[0<x<1]^{2} \cap S_{1} .
$$

The domain $V$ is compactly contained in $U$. Set $C_{5}=9 C(U, V)$, where $C(U, V)$ is the constant from Proposition 4.1 for this choice of domains $U$ and $V$. We set $V_{k, l}=V+z_{k, l}$ and $U_{k, l}=U+z_{k, l}$. By applying the previous proposition to the domains $V_{k, l}$ and $U_{k, l}$ and after taking the sum over $k, l \in \mathbf{Z}$, we obtain that inequality (33) holds. 
Lemma 4.3. Let $\left\{\phi_{n}(z)\right\}$ be the sequence given by $\left(12^{\prime}\right)$. Then $\phi_{n}(z)$ satisfies the following:

$$
\lim _{K \rightarrow \infty} \int_{\Omega_{K, n}}\left|\phi_{n}\right|=0
$$

uniformly in $n \in \mathbf{N}$. Set $\Omega_{K, n}$ is given by

$$
\Omega_{K, n}=\left\{z \in S:\left|\phi_{n}(z)\right|>K\right\} .
$$

Proof. Set

$$
s_{n}=\sup _{z \in S_{1}}\left|\phi_{n}(z)\right| .
$$

From Lemma 3.2 and in particular from inequality (19) we conclude that if $z \in \boldsymbol{\Delta}_{p, q}$ and $z \in S_{1}$ we have that $\left|\alpha_{p, q}(n)-\phi_{n}(z)\right|$ is uniformly bounded, that is the bound can be taken not to depend on a choice of pair $(p, q)$ or $n$. In particular each $s_{n}$ is a finite positive number. So we conclude that there is a constant $K_{0}$ such that $s_{n}<K_{0}$ (moreover it follows from (19) that $s_{n} \rightarrow 1, n \rightarrow \infty$ ). Therefore for $K>K_{0}$ we have that $\Omega_{k, n}$ is a subset of $S \backslash S_{1}$. In the neighborhood of the point $z_{k, l}$, the function $\phi_{n}(z)$ is of the form

$$
\phi_{n}(z)=a_{k, l}(n)(z)+\frac{\lambda_{k, l}(n)}{z-z_{k, l}}
$$

where $a_{k, l}(n)(z)$ are analytic functions and $\lambda_{k, l}(n)$ the residues of $\phi_{n}(z)$ at the points $z_{k, l}$. We now apply Lemma 4.1. We may assume that $K>3 K_{0}$. After the summation we derive the estimate

$$
\int_{\Omega_{K, n}}\left|\phi_{n}\right| \leq \frac{4 \pi}{K-2 K_{0}} \sum_{k, l}\left|\lambda_{k, l}(n)\right|^{2} .
$$

On the other hand from Lemma 4.2 and from the condition $2^{\circ}$ of Lemma 3.3 we deduce that

$$
\sum_{k, l} \int_{\frac{1}{8}<\left|z-z_{k, l}\right| \leq \frac{1}{4}} \frac{\left|\phi_{n}^{\prime}\right|^{2}}{\left|\phi_{n}\right|} \leq C_{5} \int_{S_{1}}\left|\phi_{n}\right|-R e\left(\phi_{n}\right) \leq C_{5} C
$$

holds for each $n \in \mathbf{N}$. Consider the function $d_{k, l}(n)=\left(z-z_{k, l}\right)^{4}\left(\phi_{n}^{\prime}(z)\right)^{2} . d_{k, l}(n)$ is an analytic function in $\left\{\left|z-z_{k, l}\right|<\frac{1}{2}\right\}$ and $d_{k, l}(n)\left(z_{k, l}\right)=\lambda_{k, l}(n)$. By applying the mean value theorem onto the function $d_{k, l}(n)$ and by using the fact that $\left|\phi_{n}(z)\right| \leq$ $K_{0}$ for $z \in S_{1}$, we obtain

$$
\int_{\frac{1}{8}<\left|z-z_{k, l}\right|<\frac{1}{4}} \frac{\left|\phi_{n}^{\prime}(z)\right|^{2}}{\left|\phi_{n}(z)\right|} d x d y \geq \int_{\frac{1}{8}<\left|z-z_{k, l}\right|<\frac{1}{4}} \frac{|z|^{4}\left|\phi_{n}^{\prime}(z)\right|^{2}}{\left|\phi_{n}(z)\right|} d x d y \geq \frac{\pi\left|\lambda_{k, l}\right|^{2}}{32 K_{0}} .
$$

After the summation over all $z_{k, l} \in Z$ and by using (35) we find

$$
\sum_{k, l}\left|\lambda_{k, l}(n)\right|^{2} \leq \frac{32}{\pi} K_{0} C_{5} C
$$

Now directly from the previous estimate and by applying (34) we prove the lemma. 
Proof of Theorem 1.1. We have constructed a sequence which satisfies the assumptions of Proposition 1.2. The proof follows directly from Lemma 3.3, Lemma 4.3 and Proposition 1.2.

\section{REFERENCES}

[1] G. Anderson, M.Vamanamurthy, M.Vuorinen. Conformal invariants, inequalities, and quasiconformal maps. Wiley-Interscience Publications, (1997). MR 98h:30033

[2] L.Bers, H.Royden. Holomorphic families of injections. Acta Math. 157, (1986), no 3-4, 259286 MR 88i:30034

[3] V.Božin, N.Lakic, V.Marković, M.Mateljević. Unique extremality. J. d'Analyse Math. 75 (1998), 229-338. MR 2000a:30045

[4] V.Božin, V.Marković, M.Mateljević. Unique extremality in the tangent space of the universal Teichmüller space. Integral Transforms and Special Functions 6, (1998), 145-149. MR 99d:30023

[5] C.J.Earle, I.Kra, S.Krushkal. Holomorphic motions and Teichmüller spaces. Trans. of Amer. Math. Soc. 343, (1994), 927-948. MR 94h:32035

[6] F.P.Gardiner. Teichmüller Theory and Quadratic Differentials. John Wiley and Sons, New York, (1987). MR 88m:32044

[7] J.B.Garnett. Bounded analytic functions, Pure and Applied Mathematics, (1981). MR 83g:30037

[8] S. L.Krushkal. Extremal quasiconformal mappings. Sibirsk. Mat. Zh. 10, (1969), 573-583.

[9] S.Lang. Elliptic functions. Graduate Texts in Mathematics, 112, Springer-Verlag, (1987). MR 88c:11028

[10] V.Marković. Uniquely extremal quasiconformal mappings and stationary points of the energy integral. Ph.D. Thesis, University of Belgrade (1998).

[11] V.Marković and M.Mateljević. The unique extremal QC mapping and uniqueness of HahnBanach extensions, Mat. Vesnik 48, (1996), 107-112. MR 2000c:46044

[12] M.Mateljević. The unique extremality 2. To appear in the Proceedings of Rumanian-Finish International conference (1999).

[13] C.McMullen. Amenability, Poincaré series and quasiconformal maps. Invent. Math. 97, (1989), no. 1, 95-127. MR 90e:30048

[14] O.Lehto. Univalent functions and Teichmüller spaces. Graduate Texts in Mathematics, 109, Springer, Berlin, (1987). MR 88f:30073

[15] E.Reich. Uniqueness of Hahn-Banach extensions from certain spaces of analytic functions. Math. Zeitschr. 167, (1979), 81-89. MR 80j:30074

[16] E.Reich. On criteria for unique extremality of Teichmüller mappings. Ann. Acad. Sci. Fenn. Ser. A I Math. 6, (1981), 289-301. MR 83j:30022

[17] E.Reich. The unique extremality counterexample, J. d'Analyse Math. 75, (1998), 339-347. MR 99k:30037

[18] E.Reich. Extremal problems in the unit disc. Preprint (1998).

[19] E.Reich and K.Strebel. Quasiconformal mappings of the punctured plane. Complex Analysisfifth Romanian-Finnish seminar, Part I (Bucharest 1981), Lecture Notes in Math. No. 1013, Springer Verlag (1983), 182-221. MR 85i:30045

[20] K.Strebel. On lifts of extremal quasiconformal mappings. J. d'Analyse Math. 31, (1977), 191-203. MR 58:28489

[21] K.Strebel. Extremal quasiconformal mappings. Mathematical Sciences Research Institute, Berkeley, Report No. 09419-86 August 1986, Resultate Math. 10, (1986), 168-210. MR 88a:30048

[22] K.Strebel. On the extremality and unique extremality of certain Teichmüller mappings. Complex analysis, 225-238, Birkhäuser, MA, (1988). MR 90e:30025

Institute of Mathematics, University of Warwick, Coventry, CV4 7AL, UK

E-mail address: markovic@maths.warwick.ac.uk 